\title{
A three-disulphide derivative of hen lysozyme
}

\author{
Structure, dynamics and stability
}

\author{
Sheena E. RADFORD, ${ }^{*} \dagger$ Derek N. WOOLFSON, ${ }^{*} \S$ Stephen R. MARTIN, $\ddagger$ Gordon LOWE $\dagger$ \\ and Christopher M. DOBSON*\| \\ *Inorganic Chemistry Laboratory, University of Oxford, South Parks Road, Oxford OX1 3QR, U.K., \\ $\nmid$ Dyson Perrins Laboratory, University of Oxford, South Parks Road, Oxford OX1 3QY, U.K., and \\ $\ddagger$ National Institute for Medical Research, The Ridgeway, Mill Hill, London NW7 1AA, U.K.
}

\begin{abstract}
A three-disulphide derivative of hen egg-white lysozyme was made by selective reduction and carboxymethylation of one of the four original disulphide bridges. $N$-Terminal sequencing and two-dimensional ${ }^{1} H$-n.m.r. spectroscopy revealed that the disulphide bridge linking cysteine residues 6 and 127 had been modified and that the three remaining disulphide bonds were native-like in nature. Analysis of COSY and NOESY spectra indicated that the three-disulphide lysozyme (CM ${ }^{6,127}$ lysozyme retains the same secondary and tertiary structure as its four-disulphide counterpart; its stability to $\mathrm{pH}$ and temperature is, however, dramatically decreased. N.m.r. spectroscopy was used to characterize the thermal folding and unfolding transition of $\mathrm{CM}^{6,127}$-lysozyme. Not only is the transition still a highly co-operative event, but the enthalpy change associated with folding and unfolding resembles that of intact lysozyme when their differences in thermal stability are taken into consideration. The significance of these results in terms of the folding process of lysozyme is discussed. By contrast with authentic lysozyme, $\mathbf{C M}^{6,127}$-lysozyme was found to exist in an unfolded state at $\mathrm{pH} 2$ at room temperature. N.m.r. spectroscopy and c.d. were used to characterize this state. Unlike their homologous relative, $\alpha$-lactalbumin, which exists in a partially folded molten globule state under these conditions, only residual non-native-like structure persists in the acid-unfolded state of $\mathrm{CM}^{6,127}$-lysozyme. These results indicate that the difference in folding behaviour of lysozyme and $\alpha$-lactalbumin cannot be accounted for simply by their differences in thermal stability.
\end{abstract}

\section{INTRODUCTION}

The folding of globular proteins is a complex process, beginning with a largely unstructured state and ending with a native three-dimensional structure that is functionally active. One of the major challenges of protein folding studies is the determination of the structures of folding intermediates and the elucidation of the kinetics and thermodynamics of their formation.

Although recent developments in the fields of protein engineering and n.m.r. spectroscopy have resulted in the detailed analysis of ever-increasing numbers of native protein structures in solution, very little is known about their denatured or partially folded states. Studying these states is, however, an intrinsically difficult task, since folding is usually a highly co-operative event and occurs extremely rapidly, even when retarded by the requirement for cis-trans-proline isomerism or disulphide bond formation. Thus intermediate folded states are difficult to obtain at equilibrium. One of the most detailed characterizations of folding intermediates has been achieved by trapping methods. A classic example of such an approach is seen in the sequence of disulphide bond formation in the course of oxidative refolding of reduced bovine pancreatic trypsin inhibitor (Creighton, 1978). Examination of isolated intermediate states by n.m.r. then enabled a structural basis for the folding pathway to be proposed (States et al., 1987). More recently, important advances have been made by using amide-labelling techniques coupled with two-dimensional ${ }^{1} \mathrm{H}$-n.m.r. spectroscopy to monitor the formation of regions of secondary structure early in the folding process (Roder et al., 1988; Udgaonkar \& Baldwin, 1988). Even so, relatively little is known about the detailed conformational properties of intermediate folded states or of the interactions that stabilize them, yet it is clear that such structural information is required if we wish to understand the molecular events associated with protein folding.

For certain proteins, conditions have been found under which intermediate conformational states are stable at equilibrium. The term 'molten globule' has been given to these states, which are thought to be compact molecules with similar secondary structures to the native state, but with more disordered tertiary interactions (Kuwajima, 1989). Although it has been known for some time that heat-denatured or acid-denatured proteins may not be totally devoid of residual inter-residue interactions, the first clear-cut examples of molten globule states were those of $\alpha$ lactalbumin and cytochrome $c$ at acid pH (Dolgikh et al., 1981, 1983; Ohgushi \& Wada, 1983). To date, the most elaborate structural description of the molten globule state has been provided by an n.m.r. study of this state of $\alpha$-lactalbumin (Baum et al., 1989). These results show clearly that specific regions of $\alpha$ helix persist in the molecule whereas there is no evidence for the presence of any significant $\beta$-sheet structure. Thus a model for the molten globule has arisen in which significant conformational freedom exists but where specific elements of native-like structure are preserved. The results of kinetic experiments with several proteins have shown the rapid formation of intermediates with properties consistent with a molten globule state. This suggests that the existence of the molten globule may be quite widespread and that this state may be, in general, an obligatory intermediate along the folding pathway (Kuwajima, 1989).

The unfolding transition of hen egg-white lysozyme has long

Abbreviations used : $\mathrm{CM}^{6,127}$-lysozyme, three-disulphide derivative of lysozyme carboxymethylated at residues Cys-6 and Cys-127; COSY, correlated spectroscopy; NOESY, nuclear-Overhauser-enhancement spectroscopy; n.O.e., nuclear Overhauser enhancement.

$\S$ Present address: Department of Biochemistry, University of Cambridge, Tennis Court Road, Cambridge CB2 1QW, U.K.

\| To whom correspondence should be addressed. 
been known to be a two-state co-operative event, in which only the native and unfolded states are stable at equilibrium (Imoto et al., 1972). Even so, kinetic experiments have revealed the occurrence of an early intermediate that is thought to resemble the molten globule state of its homologous relative, $\alpha$-lactalbumin (Kuwajima et al., 1985). Thus, in spite of the known differences in their equilibrium unfolding behaviour, folding of the two proteins may occur via a common structural intermediate. It has been suggested that the different equilibrium unfolding behaviours of lysozyme and $\alpha$-lactalbumin could reflect their different thermal stabilities (Kuwajima, 1989). Thus a molten globule state might not be detected in equilibrium studies of the unfolding transition of lysozyme owing to the harsh conditions required to bring about this change. The present paper describes the preparation of a derivative of lysozyme containing three native disulphide bonds and two carboxymethylated cysteine residues (6 and 127) and its detailed analysis by two-dimensional ${ }^{1}$ H-n.m.r. spectroscopy. The results show that loss of a single disulphide bond has no major effect on the three-dimensional structure of the protein, but that the stability of the enzyme is dramatically decreased. The relevance of these results in terms of the role of the Cys-6-Cys-127 disulphide bond in the formation and maintenance of secondary structure during the oxidative refolding of lysozyme and the differences in unfolding equilibria of lysozyme and $\alpha$-lactalbumin are discussed.

\section{MATERIALS AND METHODS}

\section{Preparation of $\mathbf{C M}^{6,127}$-lysozyme}

$\mathrm{CM}^{6,127}$-lysozyme was prepared by a modification of the method of Acharya \& Taniuchi (1980). A wide variety of experimental conditions was examined, but in no case was the nature of the product affected.

The following procedure was routinely adopted. Hen eggwhite lysozyme $(20 \mathrm{mg} / \mathrm{ml}$ in $0.1 \mathrm{M}$-Tris/acetate buffer, $\mathrm{pH} 7.8)$ was incubated with dithiothreitol $(5 \mathrm{mM})$ at $20^{\circ} \mathrm{C}$. The solution immediately became turbid. When the turbidity had increased 40 -fold (measured by the increase in light-scattering at $600 \mathrm{~nm}$ ), reduction was arrested by the addition of a 5-fold molar excess (over the disulphide groups of dithiothreitol) of iodoacetic acid. The reaction was allowed to proceed for $1 \mathrm{~h}$ at $20^{\circ} \mathrm{C}$. The sample was then dialysed against water at $4{ }^{\circ} \mathrm{C}$, and the soluble fraction was collected by centrifugation $\left(3000 \mathrm{~g}\right.$ for $10 \mathrm{~min}$ at $\left.4^{\circ} \mathrm{C}\right)$ and freeze-dried. $\mathrm{CM}^{6,127}$-lysozyme was purified by ion-exchange chromatography (Bio-Rex 70 column) in $0.2 \mathrm{M}$ sodium phosphate buffer, $\mathrm{pH}$ 7.1. $\mathrm{CM}^{6,127}$-lysozyme was eluted from the column in the $0.2 \mathrm{M}$ buffer described above. Unmodified lysozyme was then collected by using the same buffer, but containing $1 \mathrm{M}-\mathrm{NaCl}$. Both samples were dialysed at $4{ }^{\circ} \mathrm{C}$ against water adjusted to $\mathrm{pH} 3.8$ with $\mathrm{HCl}$ and freeze-dried.

\section{Preparation of samples for ${ }^{\mathbf{1}} \mathbf{H}$-n.m.r. spectroscopy}

Hen lysozyme (Sigma Chemical Co.) was purified by ionexchange chromatography (Bio-Rex 70 column) before use. Contaminating proteins were eluted from the column with $0.2 \mathrm{~m}$-sodium phosphate buffer, $\mathrm{pH}$ 7.1. Pure lysozyme was then collected with the same buffer, but containing $1 \mathrm{M}-\mathrm{NaCl}$. Fractions containing lysozyme were pooled, dialysed against water adjusted to $\mathrm{pH} 3.8$ with $\mathrm{HCl}$ and freeze-dried. Samples for ${ }^{1} \mathrm{H}$-n.m.r. spectroscopy were unbuffered and were $0.5 \mathrm{~mm}$ in concentration for one-dimensional experiments and $2 \mathrm{~mm}$ in concentration for two-dimensional spectroscopy. For experiments in $\mathrm{H}_{2} \mathrm{O}$ solution, samples were dissolved in $0.5 \mathrm{ml}$ of ${ }^{1} \mathrm{H}_{2} \mathrm{O} /{ }^{2} \mathrm{H}_{2} \mathrm{O}(9: 1, \mathrm{v} / \mathrm{v}$ ) and the $\mathrm{pH}$ was adjusted (usually to $\mathrm{pH} 3.8$ ) by careful addition of $\mathrm{HCl}$ or $\mathrm{NaOH}$. Dioxan (1 mM) was added as an internal reference and was taken to have a chemical shift of 3.743 p.p.m. For spectra in ${ }^{2} \mathrm{H}_{2} \mathrm{O}$ solution, the ${ }^{1} \mathrm{H}_{2} \mathrm{O} /{ }^{2} \mathrm{H}_{2} \mathrm{O}$ mixture was replaced with $0.5 \mathrm{ml}$ of $99.96 \%{ }^{2} \mathrm{H}_{2} \mathrm{O}$. For hydrogen-exchange studies the freeze-dried protein was freshly dissolved at $\mathrm{pH} 3.8$ in $99.96 \%{ }^{2} \mathrm{H}_{2} \mathrm{O}$ and spectra were acquired various times after dissolution.

\section{${ }^{1}$ H-n.m.r. spectroscopy}

N.m.r. experiments were performed at $500 \mathrm{MHz}$ on a Bruker machine equipped with an Aspect 3000 computer or on a homebuilt spectrometer equipped with an Oxford Instruments Co. magnet, a GE/Nicolet 1280 computer and a Bruker probe. All two-dimensional experiments were performed at $35^{\circ} \mathrm{C}$. Phasesensitive J-correlated spectroscopy (COSY) (Aue et al., 1976; Bax \& Freeman, 1981) was performed as described by Redfield \& Dobson (1988). Data sets consisting of $512 t_{1}$ increments of 48 transients were collected and a sweep width of $7042 \mathrm{~Hz}$ was used in each dimension. Spectra were resolution enhanced in $t_{2}$ by trapezoidal multiplication and double-exponential multiplication and in $t_{1}$ by trapezoidal multiplication only. After zero-filling twice in $t_{1}$ and once in $t_{2}$ the digital resolution was $3.5 \mathrm{~Hz} /$ point. Phase-sensitive NOESY spectroscopy was performed by published methods (Jeener et al., 1979; Anil Kumar et al., 1980). Spectra were acquired using a sweep width of $7042 \mathrm{~Hz}$ in each dimension, 256 increments and a mixing time of $200 \mathrm{~ms}$ that was randomly varied by $10 \%$. Spectra were processed as described for COSY spectra.

C.d.

Samples for study by c.d. contained $0.15 \mathrm{mg}$ of lysozyme $/ \mathrm{ml}$ in unbuffered solution at $\mathrm{pH} 3.8$ or $\mathrm{pH} 2.0$. Spectra were recorded from 260 to $180 \mathrm{~nm}$ and from 340 to $250 \mathrm{~nm}$ on a Jasco J600 spectropolarimeter in cells with $1 \mathrm{~mm}$ and $10 \mathrm{~mm}$ path lengths respectively. Spectra were collected at room temperature, unless otherwise stated. Data are reported as molar c.d. absorption coefficient $(\Delta \epsilon)$, calculated using a mean residue weight of 110 .

\section{Other methods}

Proteins were analysed by acid/urea/PAGE by a modification of the method of Moore et al. (1968). Samples $(10 \mu \mathrm{g})$ were

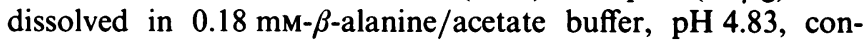
taining $8 \mathrm{M}$-urea. Gels $(7.5 \% \mathrm{~T}, 0.9 \% \mathrm{C})$ were $8 \mathrm{M}$ in urea and $0.18 \mathrm{~mm}$ in $\beta$-alanine/acetate, $\mathrm{pH} 4.83$. SDS/PAGE was performed on Tris/glycine gels $(15 \% \mathrm{~T}, 0.6 \% \mathrm{C})$ by using published methods (Laemmli, 1970). The concentration of free thiols and the number of disulphide links were determined by the method of Ellman (1959) as modified by Anderson \& Wetlaufer (1975). Automated $N$-terminal sequencing and amino acid analysis were performed by published methods (Hewick et al., 1981 ; Hendrikson \& Meredith, 1984). The catalytic activity of lysozyme was measured with Micrococcus lysodeikticus cells as described by Locquet et al. (1968).

\section{RESULTS}

\section{Characterization of $\mathrm{CM}^{6,127}$-lysozyme}

SDS/PAGE indicated that $\mathrm{CM}^{6.127}$-lysozyme and unmodified lysozyme were identical in size (Fig. 1). By contrast, during acid/urea/PAGE the proteins displayed dramatically different electrophoretic mobilities (Fig. 1). The amino acid composition of $\mathrm{CM}^{6,127}$-lysozyme was identical with that of unmodified lysozyme, except for the presence of approx. 2 mol of carboxymethylcysteine/mol of lysozyme. Thus at least one of the four disulphide bridges in lysozyme has been irreversibly opened in $\mathrm{CM}^{6.127}$-lysozyme. Furthermore, titration of $\mathrm{CM}^{6,127}$-lysozyme with Ellman's reagent in the presence of $8 \mathrm{M}$-urea showed the absence of any free thiol groups. Alkali cleavage of the remaining 


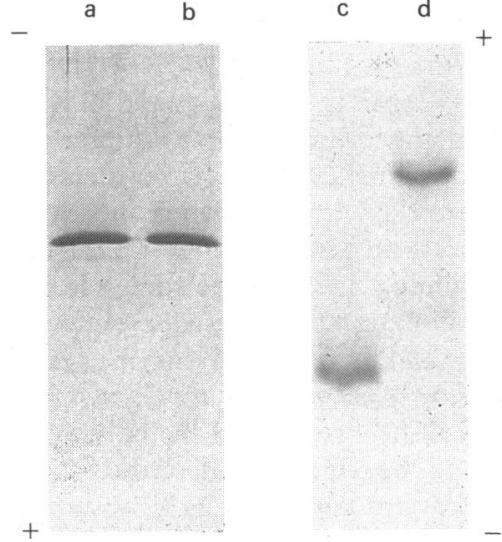

Fig. 1. SDS/PAGE (tracks a and b) and acid/urea/PAGE (tracks $c$ and d) of unmodified lysozyme (tracks $a$ and $c$ ) and $\mathrm{CM}^{6,127}$-lysozyme (tracks b and d)

The gels are stained with Coomassie Brilliant Blue. Approx. $10 \mu \mathrm{g}$ of protein was loaded on to each track on the gel.

disulphide bridges in $\mathrm{CM}^{6.127}$-lysozyme released $5.6 \mathrm{~mol}$ of thiol groups $/ \mathrm{mol}$ of protein, consistent with the presence of three intact disulphide bridges. Automated $N$-terminal sequencing indicated that Cys- 6 had been carboxymethylated. Assuming that the three remaining disulphide bridges in $\mathrm{CM}^{6,127}$-lysozyme are native-like in nature, Cys- 127 should also be carboxymethylated. This was confirmed to be the case by two-dimensional ${ }^{1}$ H-n.m.r. spectroscopy (see below). The specific catalytic activity of $\mathrm{CM}^{6.127}$-lysozyme was found to be diminished to $58 \%$ of the activity of unmodified lysozyme.

\section{Analysis of the ${ }^{1} \mathrm{H}$-n.m.r. spectrum of $\mathrm{CM}^{6,127}$-lysozyme}

The fingerprint region $\left(\mathrm{C}_{(\alpha)}-\mathrm{NH}\right)$ of the COSY spectrum of $\mathrm{CM}^{6.127}$-lysozyme is compared with that of unmodified lysozyme in Fig. 2. The spectra are remarkably similar and indicate that the overall three-dimensional structure of lysozyme has not been dramatically disrupted by loss of a single disulphide bridge. Only two cysteine resonances (Cys-6 and Cys-127) show significant differences in chemical shift in the two spectra, confirming that only one of the four disulphide bonds has been broken, that both Cys- 6 and Cys- 127 had been modified and that the remaining three disulphide bonds are native-like in nature. A plot of the chemical-shift differences as a function of the amino acid sequence (Fig. 3) indicates that the large differences are restricted to the immediate vicinity of the modification. This could arise from conformational differences in this region of the polypeptide chain, from electrostatic effects arising from the additional carboxymethyl groups, or from a combination of both of these effects. The chemical-shift movements occur both in the upfield and downfield directions and there is no systematic movement towards typical 'random-coil' chemical shifts (Wüthrich, 1986). Analysis of the NOESY spectrum confirmed that the threedimensional structure of $\mathrm{CM}^{6.127}$-lysozyme was closely similar to that of its four-disulphide counterpart. Thus, as for native lysozyme (Redfield \& Dobson, 1988), extended regions of $d_{\mathrm{NN}}$ connectivities were observed for residues 6-15, 25-38 and 89-101, corresponding to regions of $\alpha$-helix in parental lysozyme. Disruption of the disulphide bridge linking residues 6 and 127 thus has no major effect on the $\alpha$-helix encompassing residues $6-15$. In addition, extended regions of $d_{\alpha \mathrm{N}}$ connectivities were observed for residues $42-47,50-55$ and $57-60$, indicating the presence of the triple-stranded anti-parallel $\beta$-sheet. This structure was confirmed by the presence of several characteristic long-range intra-strand $d_{\mathrm{NN}}$ and $d_{\alpha \mathrm{N}}$ connectivities (Redfield \& Dobson, 1988). In addition, two long-range n.O.e.s between residue 2 and residues 39 and 40 were also visible in the NOESY spectrum. These peaks are also present in the NOESY spectrum of unmodified lysozyme (Redfield \& Dobson, 1988) and are in accord with a small anti-parallel $\beta$-sheet involving residues $1-5$ and 38-40. Clearly, modification of Cys-6 has little, if any, effect on the conformation of the $N$-terminal region of the polypeptide chain. (a) Native lysozyme

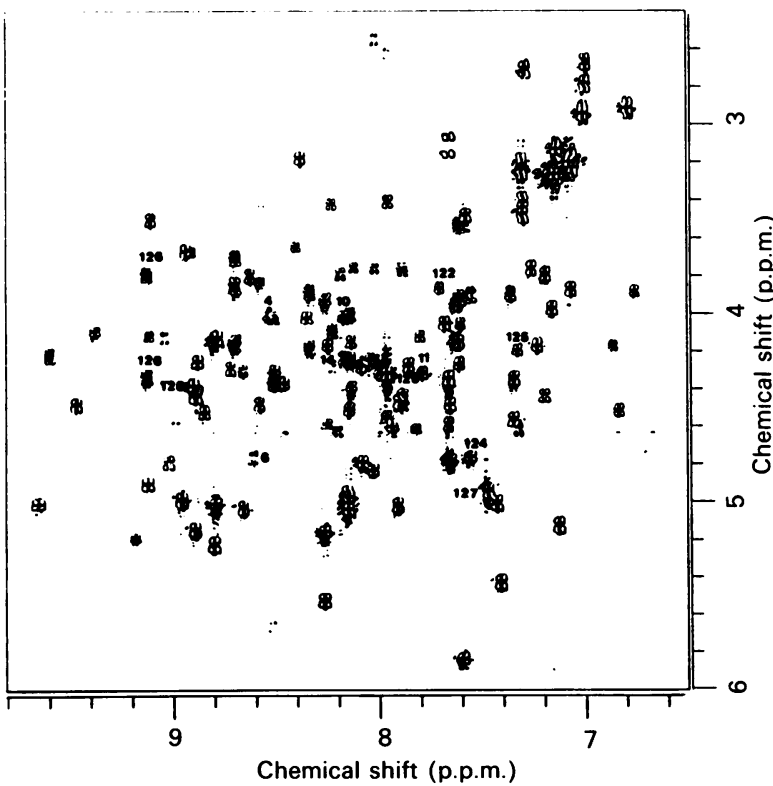

(b) $\mathrm{CM}^{6,127}$-lysozyme

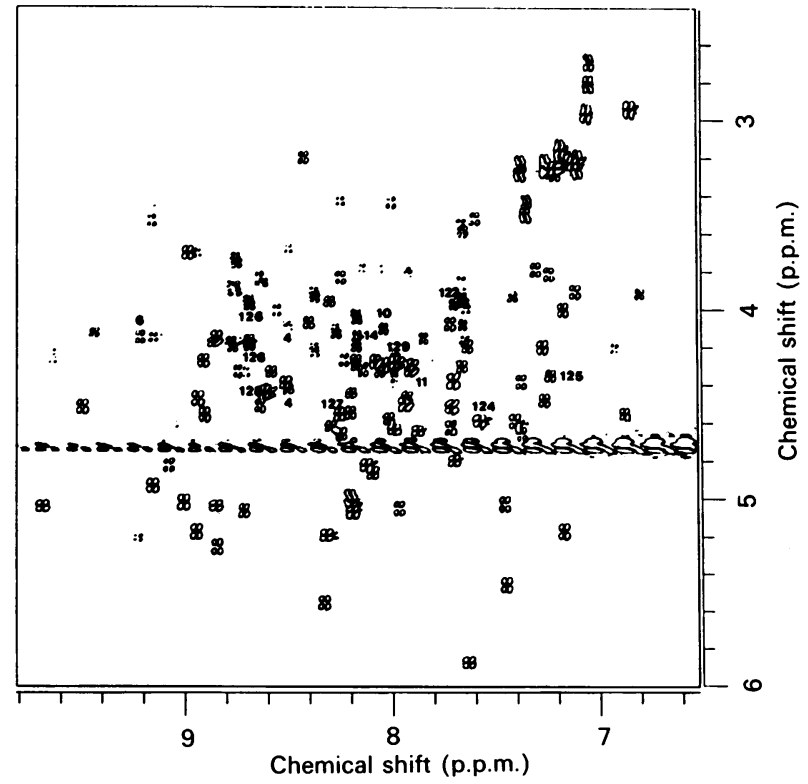

Fig. 2. Fingerprint region $\left(\mathrm{C}_{(\alpha)}-\mathrm{NH}\right)$ of the $\mathrm{COSY}$ spectra of $(a)$ unmodified lysozyme and $(b) \mathrm{CM}^{6,127}$-lysozyme

Resonances that differ significantly in chemical shift in the two spectra are labelled. Full assignment of the spectrum is given by Redfield \& Dobson (1988). The concentration of each protein sample was $2 \mathrm{mM}$. The parameters used for acquisition of the spectra are described in the Materials and methods section. 


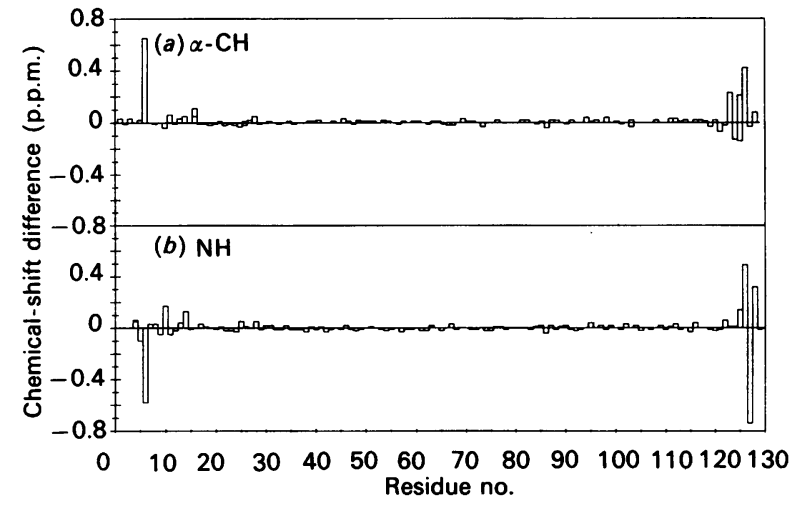

Fig. 3. Plot of the chemical-shift differences versus the amino acid sequence for lysozyme minus $\mathrm{CM}^{6,127}$-lysozyme

The $C$-terminal ten residues or so of lysozyme have little regular secondary structure, and this is reflected in a lack of extended regions of $d_{\alpha \mathrm{N}}$ or $d_{\mathrm{NN}}$ connectivities (Redfield \& Dobson, 1988). For this reason it is difficult to determine the precise structure of this region of the polypeptide chain by n.m.r. However, every sequential n.O.e. arising from the penultimate ten residues of parental lysozyme was readily identified in the NOESY spectrum of $\mathrm{CM}^{6,127}$-lysozyme (e.g. $d_{\mathrm{NN}}$ and $d_{\alpha \mathrm{N}}$ connectivities between residues $120-121,121-122,124-125,126-127$ and $127-128$ and between residues $125-126,127-128$ and 128-129 respectively). This suggests that there is no gross difference in the conformation of the $C$-terminal regions of the two proteins. To investigate this further, a search was made of the crystal structure of lysozyme for possible non-sequential n.O.e.s involving the $N$ terminal 30 residues and $C$-terminal ten residues of the polypeptide chain. Forty-two such connectivities (distances between adjacent protons of less than $0.35 \mathrm{~nm}$ ) were found, of which 13 have been assigned in the NOESY spectrum of unmodified lysozyme (C. Redfield, L. Smith \& C. M. Dobson, unpublished work). Six of these n.O.e.s are seen in the spectrum of $\mathrm{CM}^{\mathbf{6}, 127}$ lysozyme. Thus n.O.e.s connecting residues $9(\beta)-124\left(\gamma \mathrm{CH}_{3}\right)$, $26(\mathrm{~N})-121(\alpha), 26(\mathrm{~N})-120(\gamma), 27(\mathrm{~N})-120(\gamma), 29(\gamma)-123\left(\epsilon_{3}\right)$ and $30(\mathrm{~N})-120(\gamma)$ are clearly seen in the NOESY spectrum of $\mathrm{CM}^{6,127}$. lysozyme, as in that of lysozyme. By contrast, an n.O.e. between residues $6(\beta)$ and $127(\alpha)$ is observed only in the spectrum of unmodified lysozyme. An array of predicted connectivities between Leu-129 and the first $\alpha$-helix (residues 6-15) are also absent from the spectrum of $\mathrm{CM}^{6,127}$-lysozyme [6( $\left.\alpha\right)-$ $129(\delta 1,2), 9(\mathrm{~N})-129(\delta 1,2), \quad 10(\alpha)-129(\delta 1,2), 10(\mathrm{~N})-129(\delta 1,2)$, $13(N)-129(\delta 1,2)$ and $13(\beta)-129(\delta 1,2)]$. All of these cross-peaks are clearly seen in the NOESY spectrum of unmodified lysozyme acquired under identical conditions. Each of the missing n.O.e.s involves the $\delta \mathrm{CH}_{3}$ protons of the $C$-terminal residue, Leu- 129 . This observation could arise simply from movement of the side chain of Leu-129 in $\mathrm{CM}^{6,127}$-lysozyme, perhaps due to the close proximity of carboxymethyl-Cys-127. There is, therefore, some experimental evidence for conformational rearrangements in the $C$-terminal region of the polypeptide chain, although these may well be minor. To investigate this further, the possible structure of $\mathrm{CM}^{6,127}$-lysozyme was modelled by using the iterative program FRODO (Jones, 1978). The disulphide bridge linking residues 6 and 127 was removed and the side chains of the cysteine residues were modified by addition of carboxymethyl groups. The results indicated that the carboxymethyl groups could be readily accommodated in the protein structure within the constraints of the observed n.O.e.s and without alteration of the polypeptide backbone.

\section{Hydrogen-exchange properties of $\mathrm{CM}^{6,127}$-lysozyme}

$\mathrm{CM}^{6,127}$-lysozyme was freshly dissolved in ${ }^{2} \mathrm{H}_{2} \mathrm{O}$ solution at pH 3.8 and COSY spectra were acquired at $4 \mathrm{~h}$ intervals after dissolution. Unmodified lysozyme was similarly treated and the hydrogen-exchange kinetics of the two proteins were compared. Amide protons are classified into two groups: those that are not present in the spectrum acquired after $4 \mathrm{~h}$ (fast) and those with cross-peaks remaining in the spectrum $8 \mathrm{~h}$ after dissolution (slow). The overall patterns of hydrogen-exchange behaviour of $\mathrm{CM}^{6.127}$-lysozyme and unmodified lysozyme are very similar, the majority of the slowly exchanging amide groups being found in the $\alpha$-helical and $\beta$-sheet regions of the polypeptide chain. This, in conjunction with the n.O.e. data described above, confirms the overall native-like structure of $\mathbf{C M}^{6,127}$-lysozyme. There are, however, subtle differences in the two profiles. Several amide resonances lie in different exchange categories: Asn-27, Asn-37, Thr-40 and Arg-125 undergo relatively much faster exchange, whereas Arg-128 exchanges relatively more slowly than in authentic lysozyme. It must be emphasized, however, that the demarcation between the categories is somewhat arbitrary. These results may well indicate some changes in the dynamic behaviour of these residues, which are all in close proximity to the original Cys-6-Cys-127 disulphide bond. The complexity of the factors that govern hydrogen exchange, however, is such that the differences in hydrogen-exchange profiles of lysozyme and $\mathrm{CM}^{6,127}$-lysozyme are not interpreted further.

The overall rates of hydrogen exchange in lysozyme and $\mathrm{CM}^{6,127}$-lysozyme are quite different. Thus, $8 \mathrm{~h}$ after dissolution of $\mathrm{CM}^{6,127}$-lysozyme in ${ }^{2} \mathrm{H}_{2} \mathrm{O}$ solution, only about $20 \%$ of the amide protons are still visible in the spectrum. By contrast, about $50 \%$ of the amide proton resonances in lysozyme are still present in the spectrum after this time. Two distinct mechanisms for hydrogen exchange in native proteins have been identified. The first, a high-activation energy process, is associated directly with the co-operative unfolding of the protein. This mechanism has been found for lysozyme, under conditions such as those employed here, to be the dominant process for exchange of many amide hydrogen atoms even at temperatures well below the denaturation temperature (Delepierre et al., 1987). The second mechanism, with a lower activation energy, involves local fluctuations in the protein structure. The different overall hydrogenexchange rates for $\mathrm{CM}^{6,127}$-lysozyme and unmodified lysozyme may reflect predominantly the different exchange kinetics resulting from the differences in their thermal stabilities [denaturation midpoints $\left(T_{\mathrm{m}}\right)$ at $\mathrm{pH} 3.853^{\circ} \mathrm{C}$ and $77^{\circ} \mathrm{C}$ respectively]. In support of this, a linear correlation between the rates of hydrogen exchange and protein stability has been noted for the most slowly exchanging protons in bovine pancreatic trypsin inhibitor (Roder, 1989).

\section{Thermal stability of $\mathrm{CM}^{6,127}$-lysozyme}

The thermal unfolding transition of $\mathrm{CM}^{6,127}$-lysozyme was monitored by measuring the decrease in intensity of several of the resolved resonances (Leu-17 $\delta \mathrm{CH}_{3}, \mathrm{Cys}-64 \alpha \mathrm{CH}$, Ile-98 $\gamma \mathrm{CH}_{3}$ and Asn-59 $\alpha \mathrm{CH}$ ) as a function of temperature. The results are compared with those of unmodified lysozyme in Fig. 4. For both proteins the transition is clearly a highly co-operative (two-state) event. However, the thermal stability of $\mathrm{CM}^{6,127}$-lysozyme was dramatically decreased $\left(T_{\mathrm{m}} 53 \pm 2^{\circ} \mathrm{C}\right.$ in comparison with $77 \pm 2{ }^{\circ} \mathrm{C}$ for unmodified lysozyme, both at $\mathrm{pH} 3.8$ ). The equilibrium constant for the transition was measured as a function of the temperature. These data gave a van't Hoff plot to which a straight line could be fitted and from which the enthalpy at the $T_{\mathrm{m}}$ was determined to be $+327 \pm 50 \mathrm{~kJ} / \mathrm{mol}$. This value compares with an enthalpy of unfolding at the $T_{\mathrm{m}}$ for unmodified lysozyme of $+460 \pm 25 \mathrm{~kJ} / \mathrm{mol}$ (Dobson \& Evans, 1984). 


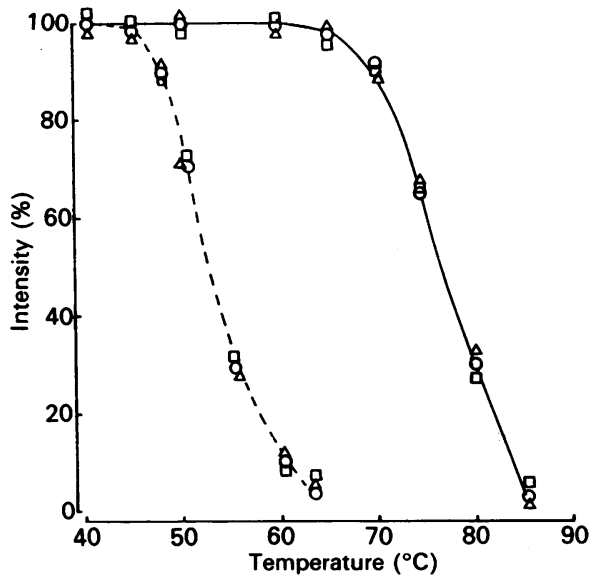

Fig. 4. Thermal unfolding of $\mathrm{CM}^{6,127}$-lysozyme

The intensities of resonances of Leu-17 $\delta \mathrm{CH}_{3}(\mathrm{O})$ Cys-64 $\alpha \mathrm{CH}(\triangle)$ and Ile-98 $\gamma \mathrm{CH}_{3}(\square)$ for lysozyme (-) and $\mathrm{CM}^{6,127}$-lysozyme $(---)$ are expressed as a percentage of their initial intensities at $35^{\circ} \mathrm{C}$. Thermal unfolding was measured with samples at $0.5 \mathrm{~mm}$ concentration; under these conditions the transition was found to be completely reversible.

\section{Characterization of the unfolded state of $\mathrm{CM}^{6,127}$-lysozyme}

Lysozyme is particularly stable to changes in $\mathrm{pH}$, and measurement of optical properties of the enzyme has shown that there is no significant change of conformation over the $\mathrm{pH}$ range 1.2-11.3 in dilute solution at moderate temperature (Imoto et al., 1972). By contrast, $\mathrm{CM}^{6,127}$-lysozyme is much more susceptible to changes in $\mathrm{pH}$, and the $\mathrm{pH}$ profile (Fig. 5) shows that the molecule exists in an unfolded state at room temperature at pH 2. Interestingly, $\alpha$-lactalbumin is also unstable under acid conditions, and at $\mathrm{pH} 2$ adopts a partially folded or molten globule state (Kuwajima et al., 1985). For this reason the acidunfolded state of $\mathrm{CM}^{6.127}$-lysozyme was examined in detail.

One of the major distinguishing features of the molten globule state is a far-u.v. c.d. spectrum very similar to that of the native state, indicating the presence of native-like secondary structure, whereas the near-u.v. c.d. spectrum indicates a denaturation-like alteration of the environment of the side chains (Kuwajima et al., 1985). The near-u.v. and far-u.v. c.d. spectra of $\mathrm{CM}^{6,127}$-lysozyme in the native, acid-unfolded and thermally unfolded states are compared in Fig. 6. As expected, the spectrum of $\mathrm{CM}^{6,127}$. lysozyme in the native state resembles closely that of authentic lysozyme, confirming the overall similarity in their threedimensional folds (Kuwajima et al., 1985). The near-u.v. and faru.v. c.d. spectra of $\mathrm{CM}^{6,127}$-lysozyme in the acid-unfolded and thermally unfolded states are also virtually identical, and are very similar to that of thermally unfolded lysozyme (Kuwajima et al., 1985). The spectra are, however, very different to that of the molten globule state of $\alpha$-lactalbumin (Kuwajima et al., 1985). Simultaneous loss of both secondary and tertiary structure in the acid-unfolded state suggests that, under these conditions, the protein does not adopt a partially folded conformation. These experiments could not be extended to higher ionic strength, which has been shown to be important in the stabilization of molten globule states of other proteins (Goto \& Fink, 1989), because of precipitation, even at the very low protein concentrations required for c.d.

The aromatic regions of the ${ }^{1} \mathrm{H}$-n.m.r. spectra of $\mathrm{CM}^{6,127}$. lysozyme in its native, thermally unfolded and acid-unfolded states are compared in Fig. 7. The wide dispersion of chemical shifts in the native state is characteristic of globular proteins and

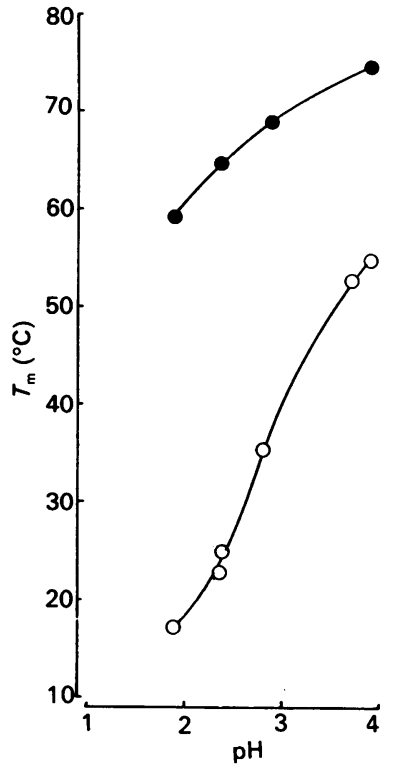

Fig. 5. Effect of pH on the stability of unmodified lysosome $(O)$ and $\mathrm{CM}^{6,127}$-lysozyme (O)

The $T_{\mathrm{m}}$ was taken to be the temperature at which the intensities of the resonances arising from the His- $15 \mathrm{H} \epsilon \mathrm{l}$ proton in the folded and unfolded states were equal. The protein concentration used for each experiment was $0.5 \mathrm{mM}$.
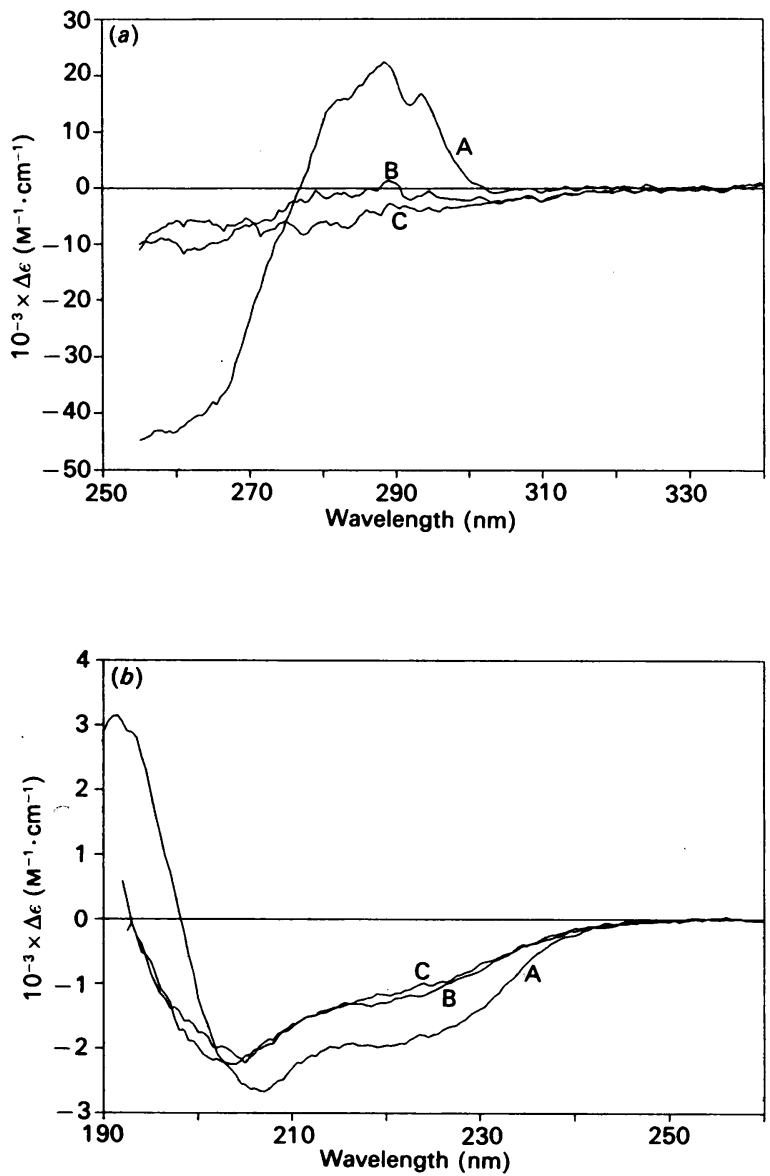

Fig. 6. (a) Near-u.v. c.d. spectra and (b) far-u.v. c.d. spectra of $\mathrm{CM}^{6,127}$ lysozyme at $\mathrm{pH} 3.8$ and $35^{\circ} \mathrm{C}$ (spectra $\mathrm{A}$ ), $\mathrm{CM}^{6,127}$-lysozyme at pH 2.0 and $35^{\circ} \mathrm{C}$ (spectra B) and $\mathrm{CM}^{\mathbf{6}, 127}$-lysozyme at $\mathrm{pH} 3.8$ and $60{ }^{\circ} \mathrm{C}$ (spectra $\mathrm{C}$ ) 

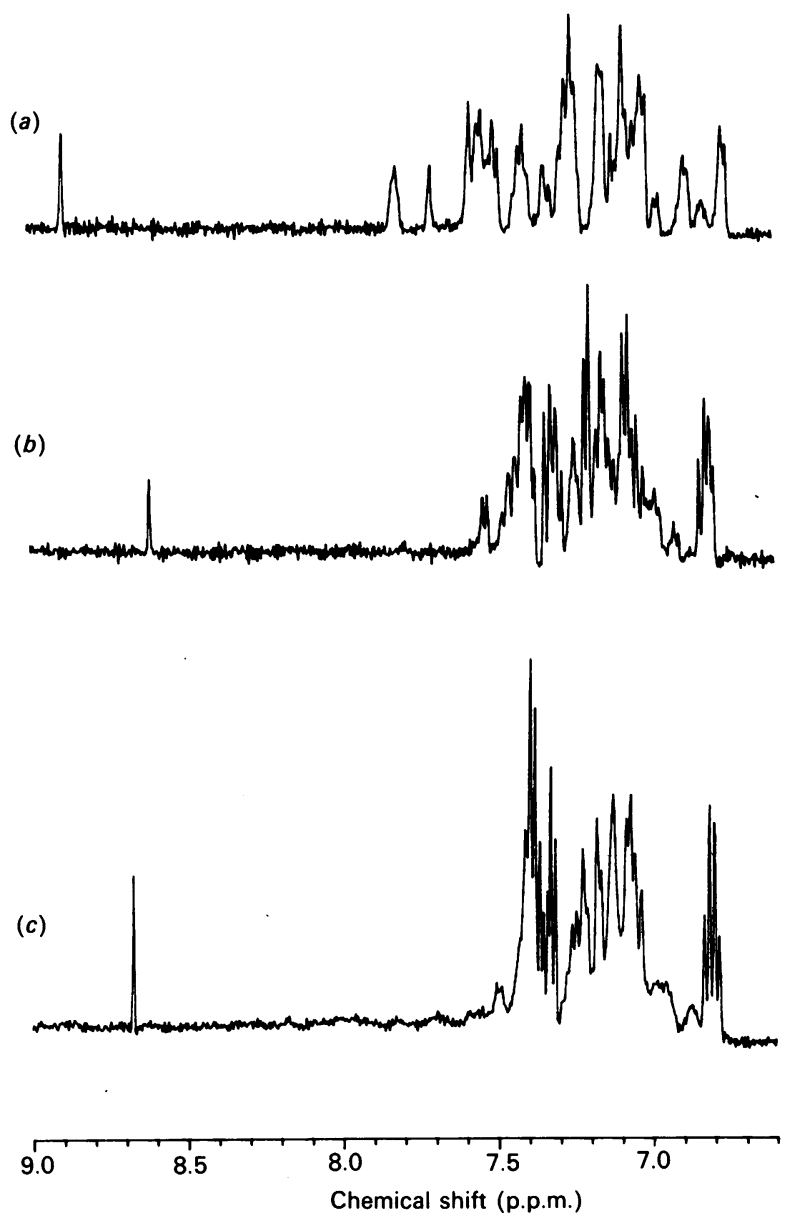

Fig. 7. Low-field region of the $500 \mathrm{MHz}{ }^{1} \mathrm{H}$-n.m.r. spectra of $\mathrm{CM}^{6,127}$ lysozyme (0.5 mM) in ${ }^{2} \mathrm{H}_{2} \mathrm{O}(a)$ at $\mathrm{pH} 3.8$ and $35{ }^{\circ} \mathrm{C}$, (b) at $\mathrm{pH} 3.8$ and $60{ }^{\circ} \mathrm{C}$ and $(c)$ at $\mathrm{pH} 2.0$ and $35^{\circ} \mathrm{C}$

reflects the inter-residue interactions within the folded structure. By contrast, the spectra of the thermally unfolded and acidunfolded states show much smaller chemical-shift dispersion, and the chemical shifts resemble more closely those predicted for an unstructured polypeptide (Wüthrich, 1986). The chemical shifts of several resonances still deviate from their predicted 'random-coil' values, indicating that residual non-random interactions still persist in the unfolded state. These interactions do not result from protein aggregation, since there is no evidence for any concentration-dependence of the ${ }^{1} \mathrm{H}$-n.m.r. spectrum, at least over the concentration range $0.5-2.0 \mathrm{mM}$. These perturbed resonances are characteristically seen in the spectrum of the thermally unfolded state of lysozyme and are thought to indicate the presence of residual non-native-like structure in the unfolded state (Evans et al., 1990). The spectra of the acid-unfolded and thermally-unfolded states of $\mathbf{C M}^{6,127}$-lysozyme are quite similar and the features characteristic of the molten-globule state of $\alpha$-lactalbumin are absent from the spectrum, i.e. significant chemical-shift dispersion and a marked broadening of several resonances (Baum et al., 1989). Clearly, the acid-unfolded and thermally unfolded states of $\mathbf{C M}^{6,127}$-lysozyme are similar and differ markedly from the molten globule state of $\alpha$-lactalbumin.

\section{DISCUSSION}

Reduction of lysozyme with low concentrations of dithiothreitol and treatment with iodoacetate traps a species that retains three of the four original disulphide bridges and contains two carboxymethylcysteine residues. Despite investigation of a wide variety of conditions, no trace of a two-disulphide species as originally reported (Acharya \& Taniuchi, 1980) was observed. Detailed two-dimensional ${ }^{1}$ H-n.m.r. spectroscopy and protein chemical analysis indicated that the disulphide link between Cys-6 and Cys-127 had been specifically opened. This conclusion is supported by a recent independent study that involved the preparation of $\mathrm{CM}^{6,127}$-lysozyme by methods similar to those used here (M. E. Denton \& H. A. Scheraga, personal communication). Loss of this single disulphide bridge had little effect on the three-dimensional structure of the protein, although the catalytic activity is decreased by about $40 \%$. Studies of folding and disulphide bond formation in reduced hen egg-white lysozyme have indicated that the process is a highly complex event (Anderson \& Wetlaufer, 1976). Many disulphide bonds are thought to accumulate during folding, but a specific detailed pathway for their formation has not yet been elucidated. It has been postulated that the first disulphide bonds to form during the GSH-catalysed regeneration of reduced lysozyme occur in the region of the protein encompassing residues 62-96 and involves cysteine residues 64, 76, 80 and 94 (Anderson \& Wetlaufer, 1976). The native disulphide bond, Cys-30-Cys-115, is thought to form late in the pathway and is followed by the formation of the Cys-6-Cys-127 disulphide link, which completes the crosslinking of the enzyme. By contrast, Acharya \& Taniuchi (1977) have reported the isolation of all four possible isomers of lysozyme containing three native disulphide bonds and one open disulphide bond. However, detailed structural information of the intermediates has not been established.

The production of only a single three-disulphide species following partial reduction of native lysozyme would be consistent with, but does not require, the proposition that during folding the Cys-6-Cys-127 disulphide bond is the last such link to form. The retention of virtually the complete three-dimensional structure of lysozyme in the absence of the Cys-6-Cys-127 disulphide bond shows that the presence of a complete set of disulphide bridges is not required for the formation of the native three-dimensional structure. This conclusion is similar to that drawn from studies of bovine pancreatic trypsin inhibitor. In this protein all three species lacking just one native-like disulphide bridge were shown to have conformations similar to that of the native protein (Stassinopoulou et al., 1984). It is noteworthy in this regard that a recombinant human lysozyme that lacks the same disulphide bridge (linking residues Cys-6 and Cys-128) is not expressed in Saccharomyces cerevisiae and is sequestered instead as an inactive and insoluble form (Taniyama et al., 1988). This suggests that either Cys-6 or Cys-128, or both, are crucial for the folding and/or secretion of human lysozyme in yeast.

Loss of a single disulphide bridge has a dramatic effect on the stability of lysozyme. The $T_{\mathrm{m}}$ of $\mathrm{CM}^{6,127}$-lysozyme is lowered by $24^{\circ} \mathrm{C}$, and at $\mathrm{pH} 2$ the protein exists in an unfolded state at room temperature (conditions under which the intact protein is fully folded). It is well known that disulphide bonds stabilize the native structure of proteins, and this effect has been attributed mainly to a decrease in the configurational entropy of the unfolded polypeptide (Creighton, 1988). A linear dependence of the denaturation enthalpy for lysozyme unfolded under different conditions with temperature has been observed (Privalov, 1989). The enthalpies of denaturation of lysozyme and $\mathrm{CM}^{6,127}$. lysozyme correlate reasonably well with their expected values based upon their different thermal stabilities. This suggests that the lower stability of $\mathrm{CM}^{6,127}$-lysozyme is probably manifested through an increased entropy loss upon protein folding, mainly through an increased configurational entropy of the unfolded state. 
It has been known for many years that lysozyme conforms to a two-state model for co-operative unfolding and that its folding transition differs significantly from that of $\alpha$-lactalbumin. At low denaturant concentrations and at $\mathrm{pH} 2$, even in the absence of denaturants, $\alpha$-lactalbumin adopts a partially folded or molten globule state in which considerable conformational order is retained (Kuwajima, 1989). A state resembling unfolded lysozyme is obtained only in the presence of high concentrations of chemical denaturants. One possible explanation for the different folding behaviours of lysozyme and $\alpha$-lactalbumin could be associated with the differences in their thermal stabilities $\left(T_{\mathrm{m}}\right.$ approx. $77^{\circ} \mathrm{C}$ and $68^{\circ} \mathrm{C}$ respectively). $\mathrm{CM}^{6.127}$-lysozyme is less stable than either of the two native proteins. As the results from n.m.r. and c.d. indicate that the acid-unfolded state of $\mathrm{CM}^{6.127}$-lysozyme is virtually completely unfolded and has no characteristics of the molten globule state of $\alpha$-lactalbumin, this does not appear to be the dominant factor in explaining the differences in folding behaviour of the two proteins.

We thank N. Soffe and J. Boyd for their assistance, C. Redfield and $\mathrm{K}$. Topping for valuable discussion, A. Willis for sequencing protein samples and M. Sutcliffe for his help with FRODO. We acknowledge the Science and Engineering Research Council for financial support. C.M.D., G.L. and S.E.R. are members of the Oxford Centre for Molecular Sciences.

\section{REFERENCES}

Acharya, A. S. \& Taniuchi, H. (1977) Proc. Natl. Acad. Sci. U.S.A. 74, 2362-2366

Acharya, A. S. \& Taniuchi, H. (1980) Int. J. Pept. Protein Res. 15, 503-509

Anderson, W. L. \& Wetlaufer, D. B. (1975) Anal. Biochem. 67, 493-502

Anderson, W. L. \& Wetlaufer, D. B. (1976) J. Biol. Chem. 251, 3147-3153

Anil Kumar, Ernst, R. R. \& Wüthrich, K. (1980) Biochem. Biophys. Res. Commun. 95, 1-6

Aue, W. P., Bartholdi, E. \& Ernst, R. R. (1976) J. Chem. Phys. 64, $2229-2246$

Baum, J., Dobson, C. M., Evans, P. A. \& Hanley, C. (1989) Biochemistry 28, 7-13

Bax, A. \& Freeman, R. (1981) J. Magn. Reson. 61, 306-320

Creighton, T. E. (1978) Progr. Biophys. Mol. Biol. 33, 231-295
Creighton, T. E. (1988) BioEssays 8, 57-63

Delepierre, M., Dobson, C. M., Karplus, M., Poulsen, F. M., States, D. J. \& Wedin, R. E. (1987) J. Mol. Biol. 197, 111-130

Dobson, C. M. \& Evans, P. A. (1984) Biochemistry 23, 4267-4270

Dolgikh, D. A., Gilmanshin, R. I., Brazhnikov, E. V., Bychkova, V. E., Semisotnov, G. V., Venyaminov, S. Y. \& Ptitsyn, O. B. (1981) FEBS Lett. 136, 311-315

Dolgikh, D. A., Abaturov, L. V., Bolotina, I. A., Brazhnikov, E. V., Bychkova, V. E., Gilmanshin, R. I., Lebedev, O. Y., Semisotnov, G. V., Tiktopulo, E. I. \& Ptitsyn, O. B. (1983) Eur. Biophys. J. 13, $109-121$

Ellman, G. L. (1959) Arch. Biochem. Biophys. 82, 70-77

Evans, P. A., Topping, K. D., Woolfson, D. N. \& Dobson, C. M. (1990) Proteins, in the press

Goto, Y. \& Fink, A. L. (1989) Biochemistry 28, 945-952

Hendrikson, R. L. \& Meredith, S. C. (1984) Anal. Biochem. 136, 65-74

Hewick, R. M., Hunkapiller, M. W., Hood, L. E. \& Dreyer, W. J. (1981) J. Biol. Chem. 256, 7990-7997

Imoto, T., Johnson, C. N., North, A. C. T., Phillips, D. G. \& Rupley, J. A. (1972) Enzymes 1, 665-867

Jeener, J., Meier, B. H., Bachmann, P. \& Ernst, R. R. (1979) J. Chem. Phys. 71, 4546-4553

Jones, T. A. (1978) J. Appl. Crystallogr. 11, 268-272

Kuwajima, K. (1989) Proteins 6, 87-103

Kuwajima, K., Hiraoka, Y., Ikeguchi, M. \& Sugai, S. (1985) Biochemistry 24, 874-881

Laemmli, U. K. (1970) Nature (London) 227, 680-685

Locquet, J. P., Saint-Blanccard, J. \& Jollès, P. (1968) Biochim. Biophys. Acta 167, 150-153

Moore, P. B., Traut, R. R., Noller, H., Pearson, P. \& Dellius, H. (1968) J. Mol. Biol. 31, 441-461

Ohgushi, M. \& Wada, A. (1983) FEBS Lett. 164, 21-24

Privalov, P. L. (1989) Annu. Rev. Biophys. Chem. 18, 47-69

Redfield, C. \& Dobson, C. M. (1988) Biochemistry 27, 122-136

Roder, H. (1989) Methods Enzymol. 176, 446-473

Roder, H., Elöve, G. A. \& Englander, W. (1988) Nature (London) 335, 700-704

Stassinopoulou, C. I., Wagner, G. \& Wüthrich, K. (1984) Eur. J. Biochem. 145, 423-430

States, D. J., Creighton, T. E., Dobson, C. M. \& Karplus, M. (1987) J. Mol. Biol. 195, 731-739

Taniyama, Y., Yamamoto, Y., Nakao, M., Kikuchi, M. \& Ikehara, M. (1988) Biochem. Biophys. Res. Commun. 152, 962-967

Udgaonkar, J. B. \& Baldwin, R. L. (1988) Nature (London) 335, 694-699

Wüthrich, K. (1986) NMR of Proteins and Nucleic Acids, p. 17, John Wiley and Sons, New York

Received 24 April 1990/11 June 1990; accepted 21 June 1990 